group patients $(P=.07)$. In patients with myocardial ischemic time $>180$ minutes, median creatine kinase-MB isotype levels were higher in the DNS group compared with the blood cardioplegia group $(P=.01)$. Clinical outcomes were similar in both groups. This suggests that for long operations, repeated delivery of a solution may be required to optimize myocardial protection. This is actually common sense and comes down to simple chemistry and physics. For a 3-hour operation, much of the potassium and lidocaine that arrests the heart will have long washed out, and operating room lights shining on the heart will heat it similar to chicken in the all-you-can-eat buffet at a local restaurant. With similar clinical outcomes, it remains uncertain if 1 solution or method of delivery is better than the other. An interesting aspect of the study is that the purported beneficial components of DNS are also present in the blood cardioplegia solution, namely magnesium sulfate (2 vs $2.5 \mathrm{~g}$ ), mannitol (3.26 vs $10 \mathrm{~g}$ ) lidocaine ( $130 \mathrm{vs} 40 \mathrm{mg}$ ), and bicarbonate (13 vs $1 \mathrm{mEq}$ ) in the DNS versus the blood cardioplegia solutions, respectively. Thus, the investigators are comparing 2 DNS-like solutions with differing concentrations of the same components. Also, 4 different surgeons were involved in the operations, and the delivery volumes were very different in the 2 groups, making direct comparisons problematic. However, this is an interesting and timely study, suggesting that DNS is safe and effective for short- and medium-length adult cardiac operations, but in the setting of a prolonged operation, surgeons should consider giving more than 1 or 2 shots of the solution. Unfortunately, with the many differences between groups, one is still left wondering which method of myocardial protection is better, or if they are in fact very similar in efficacy. After all, despite decades of investigation into different ways to protect the heart during surgery, there is still no single solution confirmed to be superior to any other. The only way to really solve this issue is to have a double-blind randomized controlled trial comparing DNS with another cardioplegic solution.

\section{References}

1. del Nido PJ, Cao-Dan H, Sommers KE, Ohkado A. Patent No. 5,407,793: An Aqueous Heart Preservation and Cardioplegia Solution. Alexandria, VA: US Patent and Trademark Office; 1995.

2. Lenoir M, Bouhout I, Jelassi A, Cartier R, Poirier N, El-Hamamsy I, et al. del Nido cardioplegia versus blood cardioplegia in adult aortic root surgery. J Thorac Cardiovasc Surg. 2021;162:514-22.e2.

\title{
Commentary: Cardioplegia in complex root surgery for adults: Many solutions, but does it really matter?
}

\section{Mustafa Zakkar, PhD, FRCS, ${ }^{\mathrm{a}}$ and Vito Domenico Bruno, $\mathrm{MD}, \mathrm{PhD}^{\mathrm{b}}$}

The importance of cardioplegia during cardiac surgery should be never undervalued: Significant improvements in cardiac surgery and complex operations have been

\footnotetext{
From the ${ }^{\mathrm{a} D e p a r t m e n t}$ of Cardiovascular Sciences, University of Leicester, Leicester, United Kingdom; and ${ }^{\mathrm{b}}$ Translational Health Science, Bristol Medical School, University of Bristol, Bristol, United Kingdom.

Disclosures: Authors have nothing to disclose with regard to commercial support.

Received for publication Jan 26, 2020; accepted for publication Jan 29, 2020; available ahead of print Feb 7, 2020.

Address for reprints: Vito Domenico Bruno, MD, PhD, Translational Health Sciences, Bristol Medical School, University of Bristol, Research Floor Level 7, Bristol Royal Infirmary, Upper Maudlin St, Bristol BS2 8HW Avon, United Kingdom (E-mail: Vito.D.Bruno@bristol.ac.uk).

J Thorac Cardiovasc Surg 2021;162:526-7

0022-5223/\$36.00

Copyright (c) 2020 by The American Association for Thoracic Surgery

https://doi.org/10.1016/j.jtcvs.2020.01.060
}

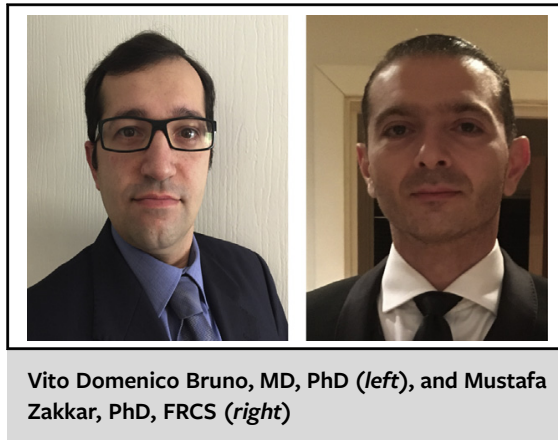

CENTRAL MESSAGE

The potential benefits of different types of cardioplegia in aortic root surgery are still debated. Del Nido cardioplegia solution may be a valid solution, although larger studies are needed. 
possible thanks to this essential solution. Traditionally, many different cardioplegia solutions have been used that can be categorized based on the nature of the solution (eg, blood vs crystalloids), the frequency of delivery (eg, single dose vs intermittent), or the temperature of the cardioplegia solution. Moreover, delivery route varies significantly and is mostly related to surgeon preference and experience. del Nido cardioplegia solution (DNS) has been in use since its development during the early 1990s for pediatric cardiac surgery. It is use has expanded into the adult cardiac surgery field and many groups report acceptable outcomes. Sorabella and colleagues ${ }^{1}$ reported their experience in redo aortic valve replacement comparing whole blood cardioplegia to DNS. The study demonstrated no differences in crossclamp (XC) or cardiopulmonary bypass (CPB) times. However, as expected, the DNS group required a significantly lower total volume of cardioplegia and retrograde cardioplegia dose. Yerebakan and colleagues, ${ }^{2}$ in 88 patients with acute myocardial infarction undergoing coronary artery bypass graft procedure, compared whole blood cardioplegia with DNS. After propensity matching, the use of DNS was associated with significantly shorter CPB and XC times. Similarly, the administration of DNS resulted in significant differences in the route and amount of delivered cardioplegia with significant reduction in total number of red blood cell units transfused and significant reduction in hospital length of stay in the DNS group. Hamad and colleagues ${ }^{3}$ reported their experience with DNS in adult combined surgery in which they compared 25 patients who underwent aortic valve replacement and coronary artery bypass graft procedure using DNS with a control group of 25 patients using whole blood cardioplegia. CPB times and XC times were lower in the DNS group. Moreover, postoperative creatine kinase, MB isotype, and troponin $\mathrm{T}$ levels were significantly lower in the DNS group; however, this did not translate to any difference in clinical outcomes. Complex aortic root interventions are highly demanding operations associated with increased ischemic time compared with conventional valve or root replacement operations. Any attempt to reduce ischemic time should be highly appreciated and using a solution that can provide adequate protection with 1 dose or a reduced number of doses that eliminates or reduces the need to stop surgery frequently to administer maintenance doses of cardioplegia is welcome. Lenoir and colleagues ${ }^{4}$ carried out a retrospective analysis reporting their experience with DNS compared with blood cardioplegia. Between 2015 and 2018, 283 consecutive patients undergoing Ross procedure $(\mathrm{n}=204)$ and valve-sparing operation $(\mathrm{n}=79)$ were included. The unmatched population included $130 \mathrm{pa}-$ tients who received DNS and 153 who received blood cardioplegia. The matched cohort included 50 patients in each arm. The primary outcome studied was myocardial injury during the first 48 hours after surgery, assessed by recording the highest postoperative creatine kinase-MB (CK-MB) and troponin T levels as well as incidence of myocardial infarction (defined as postoperative CK-MB $>100 \mu \mathrm{g} / \mathrm{L}$ ), return to spontaneous rhythm, use of inotropic support, and postoperative left ventricular ejection fraction assessed by transthoracic echocardiography before discharge. The secondary outcomes included mortality and morbidity. The analysis demonstrated that the median postoperative CK-MB peak level was similar in the DNS and blood cardioplegia groups $(48.9 \mu \mathrm{g} / \mathrm{L}$ for DNS vs $51.2 \mu \mathrm{g} / \mathrm{L}$ for blood cardioplegia; $P=.19$ with quantile regression). However, in patients with long $\mathrm{XC}$ times (myocardial ischemia $>180$ minutes) postoperative CKMB levels were significantly higher in the DNS group (75.1 $\mu \mathrm{g} / \mathrm{L}$ for DNS vs $60.5 \mu \mathrm{g} / \mathrm{L}$ for blood cardioplegia; $P=.047)$. The incidence of myocardial infarction, use of inotropic support and predischarge left ventricular ejection fraction (although too early to draw any conclusions) were similar in the DNS and blood cardioplegia groups; however, return to spontaneous sinus rhythm while weaning CPB was observed more frequently in the DNS group. Moreover, mean $\mathrm{CPB}$ time and $\mathrm{XC}$ times were significantly shorter in the DNS group. But there were no differences in perioperative mortality, morbidity, or blood transfusion requirements. The study has many limitations, as is expected with retrospective single-center studies, but the researchers tried to minimize bias by propensity matching the groups. An in-house blood cardioplegia solution was compared with DNS, which limits generalizability of results to other centers. However, it demonstrates that the use of DNS in adults undergoing complex aortic root surgery can provide comparable clinical outcomes to blood cardioplegia, and it adds more to our understanding of DNS in adults.

Finally, there are many different single-dose solutions used in complex valve surgery, many of which have been used by some of the major aortic groups around the world with satisfactory results. Thus, it will be of more interest to compare DNS with solutions such as Custodiol HTK Solution (Essential Pharmaceuticals LLC, Durham, NC) rather than with blood cardioplegia.

\section{References}

1. Sorabella RA, Akashi H, Yerebakan H, Najjar M, Mannan A, Williams MR, et al. Myocardial protection using Del Nido cardioplegia solution in adult reoperative aortic valve surgery. J Card Surg. 2014;29:445-9.

2. Yerebakan H, Sorabella RA, Najjar M, Castillero E, Mongero L, Beck J, et al. De Nido cardioplegia can be safely administered in high-risk coronary artery bypass grafting surgery after acute myocardial infarction: a propensity matched comparison. J Cardiothorac Surg. 2014;9:141.

3. Hamad R, Nguyen A, Laliberte E, Bouchard D, Lamarche Y, El-Hamamsy I, et al Comparison of Del Nido cardioplegia with blood cardioplegia in adult combined surgery. Innovations (Phila). 2017;12:356-62.

4. Lenoir M, Bouhout I, Jelassi A, Cartier R, Poirier N, El-Hamamsy I, et al. del Nido cardioplegia versus blood cardioplegia in adult aortic root surgery. J Thorac Cardiovasc Surg. 2021;162:514-22.e2. 